\title{
Effect of 20-hydroxyeicosatetraenoic acid on biological behavior of human villous trophoblasts and uterine vascular smooth muscle cells
}

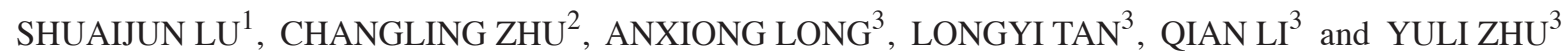 \\ Departments of ${ }^{1}$ Clinical Laboratory and ${ }^{2}$ Gynaecology and Obstetrics, The Affiliated Hospital of Ningbo University, \\ Ningbo, Zhejiang 315000; ${ }^{3}$ Department of Clinical Laboratory, Baoshan Branch of \\ Shanghai First People's Hospital, Shanghai 200940, P.R. China
}

Received October 3, 2013; Accepted February 12, 2014

DOI: $10.3892 / \mathrm{mmr} .2014 .2017$

\begin{abstract}
During pregnancy, disorders in uterine spiral artery remodeling (USAR) cause preeclampsia and other diseases. The aim of this study was to investigate the effect of 20-hydroxyeicosatetraenoic acid (20-HETE) on the biological behavior of human villous trophoblasts (HVTs) and human uterine vascular smooth muscle cells (HUVSMCs), and explore the role of 20-HETE in USAR. 20-HETE and its inhibitor HET0016 were used to study migration, invasion and apoptosis in the HVT and HVT-HUVSMC models. 20-HETE inhibited the migration and invasion of HVTs, and inhibited apoptosis in HUVSMCs and HUVSMCs co-cultured with HVTs. 20-HETE had thus obvious effects on the biological behavior of HVTs and HUVSMCs. These effects may cause USAR disorders and vascular dysfunction, leading to preeclampsia.
\end{abstract}

\section{Introduction}

The pathogenesis of preeclampsia (PE) is complex, involving immunological, genetic and nutritional factors (1), but the above factors can not fully explain its clinical and pathophysiological behavior. However, numerous studies have found that there are similar pathophysiological characteristics in cases of preeclampsia: namely, the disorders of uterine spiral artery remodeling (USAR) cause placental ischemia and hypoxia, followed by the release of a high number of vasoactive factors into the peripheral circulation. The vascular endothelial functions are thus impaired, leading to the appearance of various clinical PE symptoms, with the USAR disorder being the central

Correspondence to: Dr Longyi Tan, Department of Clinical Laboratory, Baoshan Branch of Shanghai First People's Hospital, Shanghai 200940, P.R. China

E-mail: longyitancn@126.com

Key words: 20-hydroxyeicosatetraenoic acid, uterine spiral artery remodeling, preeclampsia, human villous trophoblasts, human uterine vascular smooth muscle cells one (2). The mechanisms causing USAR disorder are numerous, but none has been elucidated yet. Studies focusing on the underlying mechanisms of USAR disorder have gained considerable interest in the context of elucidating PE pathogenesis.

Spiral artery is the branch of the uterine artery that mainly provides nutrients to the uterine basement membrane. Since 1967, when Brosens et al (3) first proposed the concept of 'spiral artery remodeling' (SAR), the roles of the spiral artery in pregnancy have gradually attracted the scientific attention. Gestational SAR is a highly complex process, mainly mediated by the trophoblast mother cells (4). With the progress of pregnancy, ectodermal trophoblast mother cells differentiate into two types: The syncytiotrophoblasts and the extravillous trophoblast cells (ETCs). ETCs invade the spiral arteries via their extremity and against the blood flow direction, as well as spiral arteries surrounding the decidual stroma $(5,6)$. The villous trophoblast cells inside and outside the spiral arteries interact with endothelial and smooth muscle cells, mediate their apoptosis, secrete the matrix metalloproteinase (MMP) protein to degrade the elastic matrix of the spiral arterial walls, and secrete an amorphous cellulose-like substance (endotheliocyte/uterine smooth muscle cell/decidua) to replace it at the same time, until the ETCs and the cellulose-like substance become the main component of the spiral arterial wall (5-7). The diameter of the remodeled spiral arteries increases, and their sensitivity towards maternal vasomotor regulatory factors is reduced, forming vessels with high flow rate and low resistance to provide sufficient nutrient and oxygen supply for fetus and placenta (6). Any alteration in the events of this process can lead to SAR disorder, followed by pregnancy complications such as PE, and restricted fetal growth.

A number of studies has found that 20-hydroxyeicosatetraenoic acid (20-HETE), one of the arachidonic acid metabolites, is the upstream regulatory factor or the $2 \mathrm{nd} / 3 \mathrm{rd}$ messenger of multiple signal transduction systems (e.g., HIF-1 $\alpha$ and $\mathrm{PI} 3 \mathrm{~K} / \mathrm{AKT}$ ) involved in the regulation of cell proliferation, differentiation, apoptosis and migration in vascular endothelial and smooth muscle cells (8-10). Therefore, 20-HETE can be considered as a key factor in functional regulation, and abnormalities related to its metabolism are expected to result in dysfunctions of endothelial and smooth muscle cells, leading 
to vascular dysfunctional diseases, such as hypertension (11). Llinás et al (12) found that pregnant rats with chronic uteroplacental ischemia exhibited certain PE features. The authors confirmed that these rats have a reduced 20-HETE production due to the inhibition of the activity of the cytochrome P450 that catalyzes its formation, and show reduced hypertension, induced by reductions in placental perfusion pressure. These results indicated that 20-HETE is involved in the development of vascular disorders, and may be involved in the regulation of placental vessels in pregnant rats (5).

In the present study, we hypothesized, based on the relevant characteristics of PE and gestational USAR, that since 20-HETE is the upstream regulatory factor of multiple signal transduction pathways involved in the functional maintenance of vascular endothelial and smooth muscle cells, abnormalities in its metabolism would result in altered transduction of the related signal, thus leading to SAR and vascular dysfunction disorders, and causing preeclampsia. In order to prove this hypothesis, mono- or co-cultures of the main cells involved in SAR (human villous trophoblasts, HVTs and human uterine vascular smooth muscle cells, HUVSMCs) were treated with 20-HETE and its inhibitor (HET0016). Using this model, we investigated the effect of 20-HETE on apoptosis of HUVSMCs co-cultured with HVTs, which was caused by the invasion of 20-HETE into the villous trophoblasts, as well as the effect of 20-HETE on USAR, with the aim of gaining novel insights on the pathogenesis of PE. To the best of our knowledge, this is the first report on a similar study system.

\section{Materials and methods}

Establishment of the co-culture model. The cultured HVT (ScienCell Research Laboratories, Carlsbad, CA, USA) and HUVSMCs (PromoCell GmbH, Heidelberg, Germany) were all adherent-growing cells, and the culture system was Dulbecco's Modified Eagle's Medium/F12 (Gibco-BRL, Carlsbad, CA, USA). The $3.0 \mu \mathrm{m}$ aperture Transwell chamber was placed into the 24-well plate and the hydration was performed in a $5 \% \mathrm{CO}_{2}$ and $37^{\circ} \mathrm{C}$ incubator for $2 \mathrm{~h}\left(\mathrm{CO}_{2}\right.$ incubator; Forma Scientific Inc., Marietta, OH, USA). The HVT and HUVSMC cells in the logarithmic growth phase were collected, then the cell suspension density was $1 \times 10^{5}$ cells $/ \mathrm{ml}$ and $1 \times 10^{5}$ cells $/ \mathrm{ml}$ with the prepared optimized concentrations of the corresponding drugs that could generate the best culture environment. The HVT cell suspension $(200 \mu 1)$ was added into the upper chamber, and the lower chamber was added with $500 \mu 1$ HUVSMC cell suspension, which was then placed in the $5 \% \mathrm{CO}_{2}$ and $37^{\circ} \mathrm{C}$ incubator for $48 \mathrm{~h}$ cultivation, followed by the corresponding detection. The co-culture model was based on that of Stec et al (13).

Determination of optimal drug concentration and treatment time. HVTs and HUVSMCs were treated with a gradient of concentrations of 20-HETE and its inhibitor HET0016 (Cayman Chemical Co., Ann Arbor, MI, USA) with 5 replicates for each concentration. The optimal drug concentration and treatment time were determined based on the results of the MTT assay (Sigma-Aldrich, St. Louis, MO, USA), used to detect the proliferation of cells after 24 and $48 \mathrm{~h}$ (Microplate reader; Bio-Rad, Hercules, CA, USA).
Assessment of HVT invasive ability and MMP-2 expression. HVTs in logarithmic growth phase were collected. The cell suspension density was adjusted using media containing the drug at the optimal concentration. Five wells were set for each group. The experiment was conducted as follows: i) the cell suspension was added to a Transwell chamber (Nunc A/S Plastfabrikation, Roskilde, Denmark) having a well diameter of $8.0 \mu \mathrm{m}$. The cells were incubated at $37^{\circ} \mathrm{C}$, under $5 \% \mathrm{CO}_{2}$ for $48 \mathrm{~h}$, followed by staining with $0.1 \%$ crystal violet staining (Beyotime Institute of Biotechnology, Shanghai, China) and determination of the HVT invasive ability according to a previously described method (13). ii) The cell suspension was added to a 24 -well plate. Following incubation $\left(37^{\circ} \mathrm{C}, 5 \% \mathrm{CO}_{2}\right)$ for $48 \mathrm{~h}$, the total RNA of cells was extracted, followed by determination of the MMP-2 mRNA level using a RT-PCR kit (TRIzol Reagent Power SYBR ${ }^{\circledR}$ Green PCR Master mix; Invitrogen, Carlsbad, CA, USA). iii) The cell suspension was added to a 24-well plate. Following incubation $\left(37^{\circ} \mathrm{C}, 5 \% \mathrm{CO}_{2}\right)$ for $48 \mathrm{~h}$, the cell supernatant was collected, and the protein concentration of MMP-2 was detected by ELISA (SuperScript $^{\circledR}$ Vilo $^{\text {TM }}$ cDNA Synthesis kit; R\&D Systems Inc., Minneapolis, MN, USA). Two-step immunohistostaining was performed, using rat anti-human monoclonal anti-MMP-2 (Santa Cruz Biotechnology, Inc., Santa Cruz, CA, USA) as the primary antibody and goat anti-rabbit HRP-polymer IgG (Beyotime Institute of Biotechnology) as the second antibody. The scoring was conducted using a double-blind method as in (14).

Determination of HUVSMC apoptotic rate. HVTs/HUVSMCs in logarithmic growth phase were collected. The cell suspension density was adjusted using media containing the drug at the optimal concentration. Five wells were set for each group. HVTs and HUVSMCs were added to the upper and lower Transwell chamber (well diameter, $3.0 \mu \mathrm{m}$ ), respectively. Following incubation $\left(37^{\circ} \mathrm{C}, 5 \% \mathrm{CO}_{2}\right)$ for $48 \mathrm{~h}$, HUVSMCs was collected. The apoptotic rate of HUVSMCs was determined using AnnexinV-fluorescein isothiocyanate (FITC), (Sigma-Aldrich), the Annexin V-FITC Apoptosis Detection kit (Sigma-Aldrich) and a flow cytometer (Beckman Coulter, Miami, FL, USA).

Statistical analysis. The SPSS 17.0 software package (IBM, Armonk, NY, USA) was used to statistically analyze the experimental data. Intergroup comparisons were performed with single- or multi-factor analysis of variance, and LSD (when data met the condition of homogeneity of variance) and Dunnett's (when data did not meet the condition of homogeneity of variance) tests were used to assess the significance of differences, with $\mathrm{P}<0.05$ indicating a significant difference.

\section{Results}

Optimal drug concentration and treatment time. Following 24and 48-h treatment of HVTs and HUVSMCs with increasing concentrations of 20-HETE and/or HET0016, the MTT assay was used to detect cell proliferation (by measuring OD values). The multi-factor analysis of variance revealed that the effect of $10 \mathrm{ng} / \mathrm{ml}$ of 20-HETE on HVT and HUVSMC proliferation was most prominent after $48 \mathrm{~h}$ of incubation. This concentration of 20-HETE enhanced the proliferation of both cell types (Fig. 1A). As for HET0016, the most prominent effect on HVT and HUVSMC proliferation was observed after $48 \mathrm{~h}$ of 
A

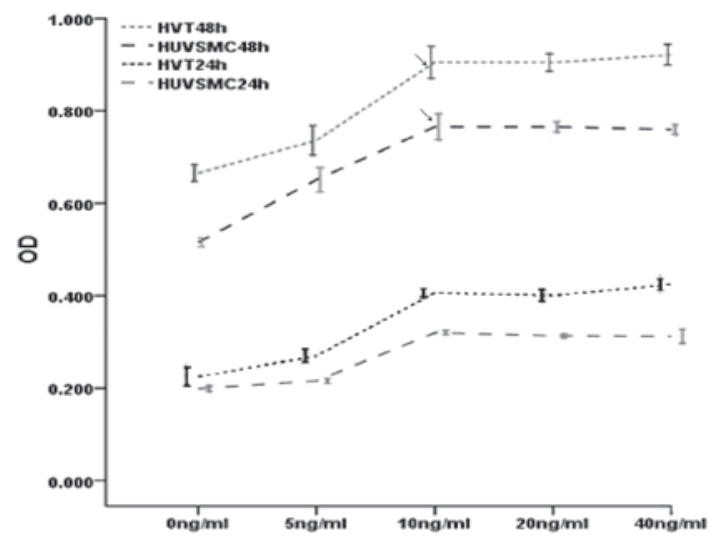

B

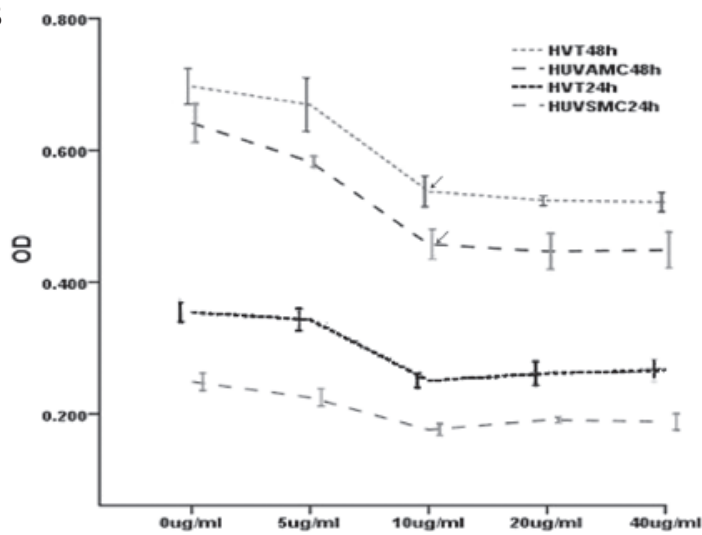

Figure 1. Effects of different treatment periods and concentrations of (A) 20-hydroxyeicosatetraenoic acid (20-HETE) and (B) HET0016 on the proliferation of human villous trophoblasts (HVTs) and human uterine vascular smooth muscle cells (HUVSMCs). Multivariate analysis of variance showed that treatment (48 h) with $10 \mathrm{ng} / \mathrm{ml}$ of 20-HETE enhanced the proliferation of HVTs and HUVSMCs $(\mathrm{P}<0.05)$, while treatment (48 h) with $10 \mu \mathrm{g} / \mathrm{ml} \mathrm{HET0016}$ inhibited the proliferation of HVTs and HUVSMCs $(\mathrm{P}<0.05)$. Arrows indicate significant differences.

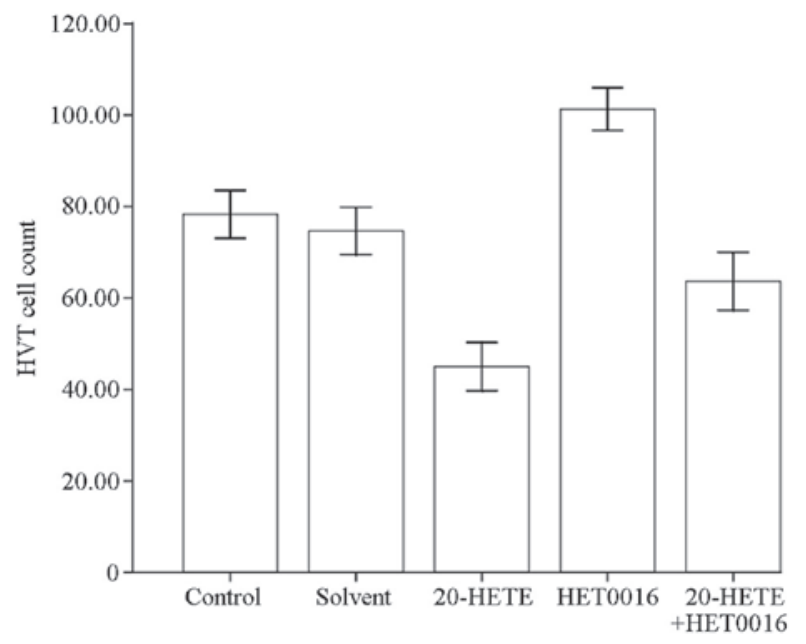

Figure 2. Effects of 20-hydroxyeicosatetraenoic acid (20-HETE) and HET0016 on the number of invading human villous trophoblasts (HVTs). Compared to the control group, the number of invading HVTs in the Transwell chamber assay was reduced in the 20-HETE group and the 20-HETE + HET0016 group, while it was increased in the HET0016 group.

incubation with $10 \mu \mathrm{g} / \mathrm{ml}$ of the inhibitor; at this concentration, the proliferation of both cell types was inhibited (Fig. 1B).

Inhibitory effect of 20-HETE on the invasive ability of HVTs. The Transwell chamber assay combined with trypan blue staining was performed to study the invasive ability of HVTs after $48 \mathrm{~h}$ of treatment with 20-HETE and HET0016. HVT cells were counted in 5 non-overlapping fields of the optical microscope (Olympus BX41, Tokyo, Japan; magnification, $\mathrm{x} 400$ ). Compared to the control group (normal group without drug and reagents), no statistically significant difference was observed in the solvent group $(\mathrm{P}>0.05)$, while the number of invading HVTs was significantly reduced $(\mathrm{P}<0.05)$ after 20-HETE treatment, was significantly increased after HET0016 treatment $(\mathrm{P}<0.05)$, and was significantly reduced $(\mathrm{P}<0.05)$ after 20-HETE + HET0016 treatment (Fig. 2).

Effect of 20-HETE on MMP-2 expression. Following treatment of HVTs with 20-HETE and HET0016 for 48 h, cDNA was synthesized from total cellular RNA, the MMP-2 gene was amplified by PCR and the product was run on an agarose gel, in order to detect its relative expression using a gel imaging system. No significant difference between the solvent and the control group ( $\mathrm{P}>0.05$ ) was observed, while the expression of MMP-2 was significantly reduced in the 20-HETE group $(\mathrm{P}<0.05)$, significantly increased in the HET0016 group $(\mathrm{P}<0.05)$, and significantly reduced $(\mathrm{P}<0.05)$ in the 20-HETE + HET0016 group compared with the control (Fig. 3A).

Following HVT treatment with 20-HETE and HET0016 for $48 \mathrm{~h}$, the supernatant was collected for the detection of the MMP-2 protein using an ELISA assay. No significant difference between the solvent and the control group ( $>>0.05$ ) was observed, while the protein levels of MMP-2 were significantly reduced in the 20-HETE group $(\mathrm{P}<0.05)$, significantly increased in the HET0016 group $(\mathrm{P}<0.05)$, and significantly reduced $(\mathrm{P}<0.05)$ in the 20-HETE + HET0016 group compared with the control (Fig. 3B). MMP-2 was also detected with immunohistostaining following 20-HETE and HET0016 treatment of HVTs for $48 \mathrm{~h}$. No difference in detected MMP-2 was observed in the comparison between the 20-HETE, the 20-HETE + HET0016 and the solvent group to the control, while the expression of MMP-2 was prominently detected in the HET0016 group (Fig. 3C).

Inhibition of HUVSMC apoptosis by 20-HETE. Following HUVSMC treatment with 20-HETE and HET0016 for $48 \mathrm{~h}$, Annexin-V FITC staining and flow cytometric analysis were performed to estimate the apoptotic rate (\%) of HUVSMCs. No statistically significant difference was observed between the solvent and the control group ( $\mathrm{P}>0.05)$, while the apoptotic rate was significantly reduced in the 20-HETE group $(\mathrm{P}<0.05)$, significantly increased in the HET0016 group $(\mathrm{P}<0.05)$, and significantly reduced $(\mathrm{P}<0.05)$ in the 20-HETE + HET0016 group compared to the control (Fig. 4A). The same analyses were performed to detect the apoptotic rate of HUVSMCs following treatment of the HVT-HUVSMC co-culture with 20-HETE and HET0016 for $48 \mathrm{~h}$. No statistically significant difference was observed between the solvent and the control group $(\mathrm{P}>0.05)$, while the apoptotic rate was significantly reduced in the 20-HETE group $(\mathrm{P}<0.05)$, significantly 
A

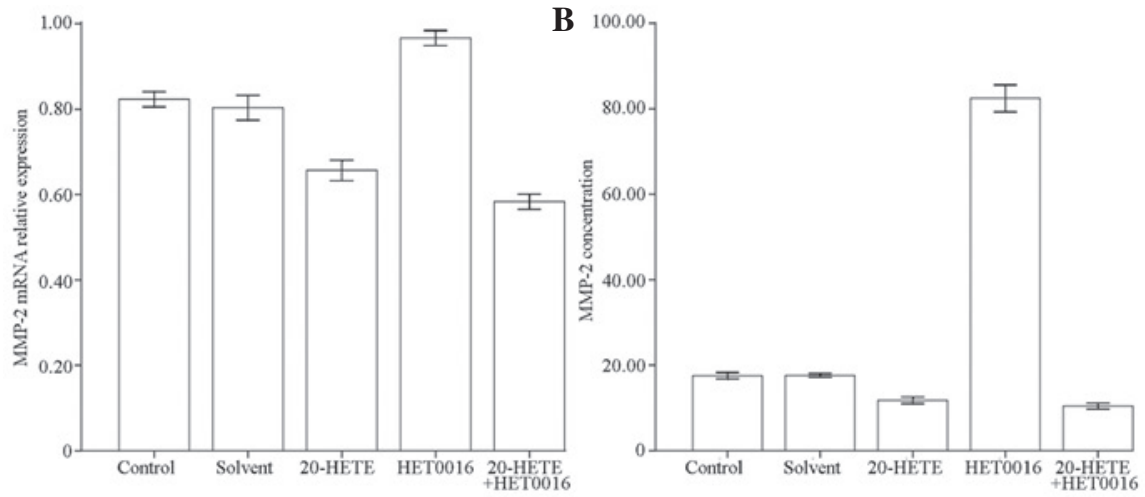

C
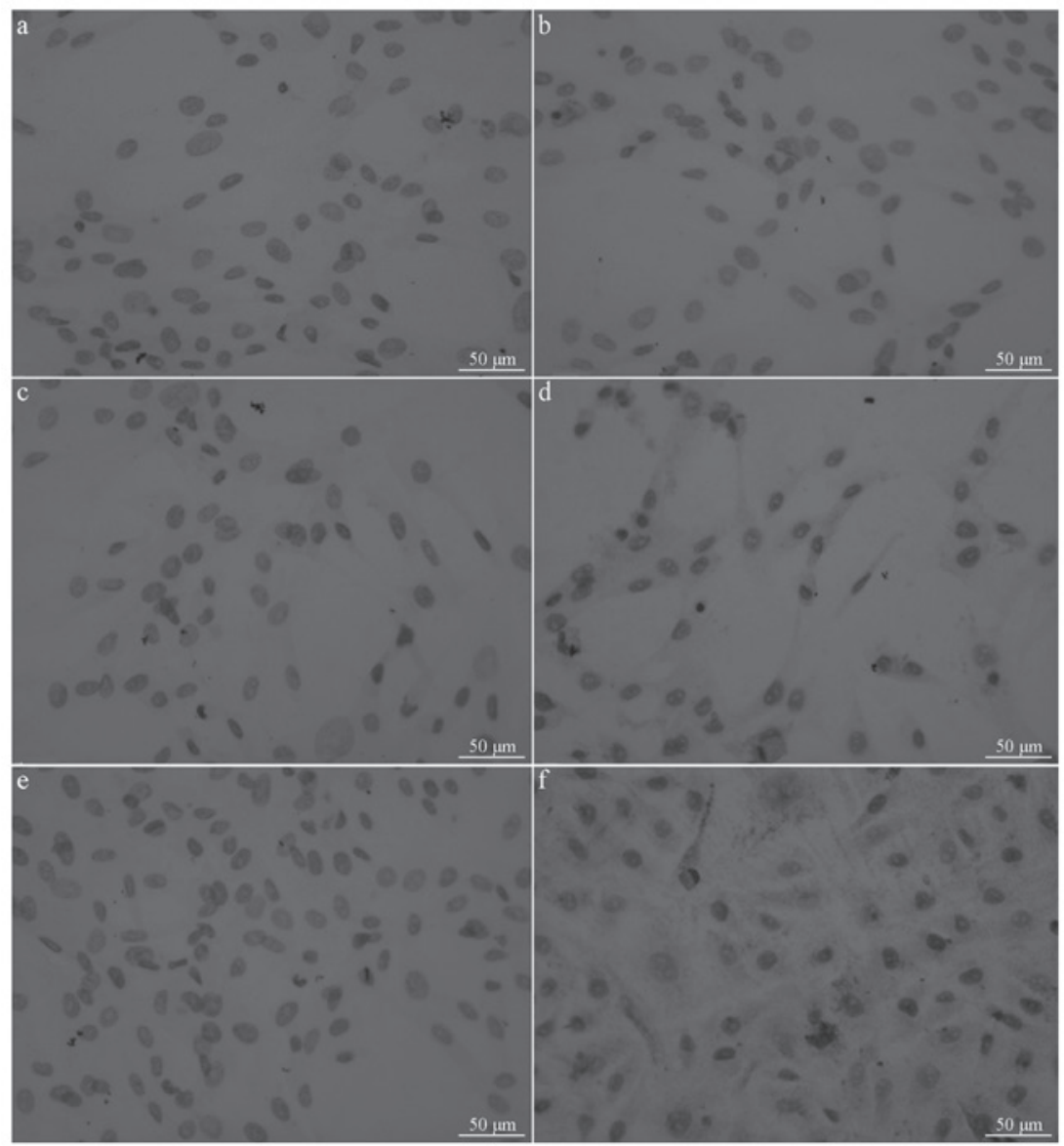

Figure 3. Effects of 20-hydroxyeicosatetraenoic acid (20-HETE) and HET0016 on (A) the mRNA expression of matrix metalloproteinase 2 (MMP-2) in human villous trophoblasts (HVTs) and (B) MMP-2 protein concentration (ng/ml) in the HVT supernatant. (C) Immunohistochemical detection of MMP-2 in 20-HETE- and HET0016-treated HVTs (magnification, x200). Compared to the control group, the transcriptional level, the protein concentration in the supernatant and the expression of MMP-2, as assessed by immunohistochemistry, are reduced in the 20-HETE group and the 20-HETE + HET0016 combined treatment group, while the above indexes are increased in the HET0016 group.

increased in the HET0016 group $(\mathrm{P}<0.05)$, and significantly reduced $(\mathrm{P}<0.05)$ in the 20 -HETE + HET0016 group compared to the control (Fig. 4B).

\section{Discussion}

Arachidonic acid (AA) is metabolized into hydroxyeicosatetraenoic acids (HETEs) and epoxy eicosatriene acids (EETs) by cytochrome P450 (CYP450) proteins (15). In the human body, the two main CYP450 isoenzymes are the CYP 4A and $4 \mathrm{~F}$, metabolizing AA into 20-HETE (16), while the other two isoenzymes, CYP 2C and 2J, metabolize AA into EETs (17). HET0016 is a specific inhibitor of 20-HETE, which can selectively inhibit the in vivo activities of CYP $4 \mathrm{~A}$ and $4 \mathrm{~F}$, while increased doses of HET0016 can also inhibit CYP 2C and 2J (18). The active concentrations of 20-HETE and HET0016 depend on the type of tissue and cell. There is currently no report on the impact of 20-HETE on the main cells involved in USAR, HVTs and HUVSMCs. In the present study, 20-HETE and its inhibitor HET0016 were used to treat HVTs and HUVSMCs, and the resulting changes in the biological behavior of these cells are discussed below.

In order to identify the most potent effects of 20-HETE and HET0016 on HVTs and HUVSMCs, we referred to the reported active concentrations of 20-HETE and HET0016 in the endothelial cells and the smooth muscle cells, and studied the effects 
A

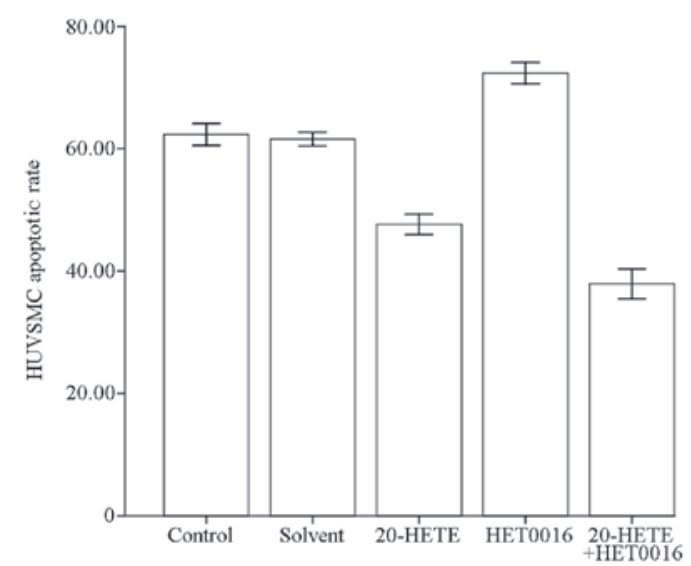

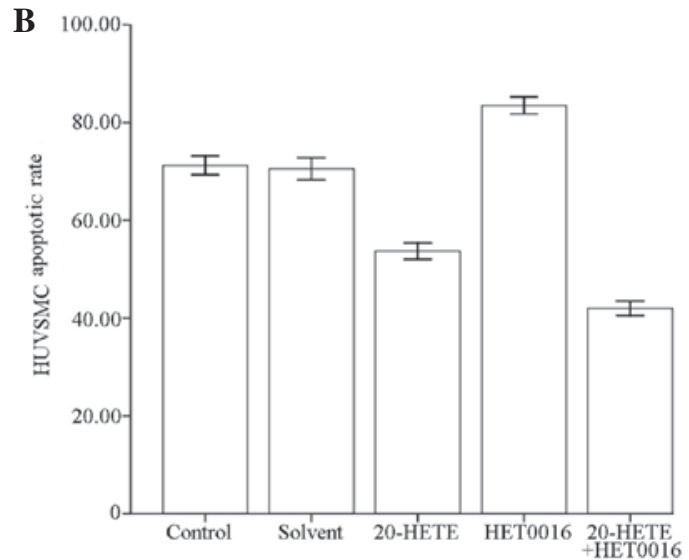

Figure 4. Effects of 20-hydroxyeicosatetraenoic acid (20-HETE) and HET0016 on apoptosis of human uterine vascular smooth muscle cells (HUVSMCs) in (A) monoculture and (B) in HVT-HUVSMC co-culture. Compared to the control group, HUVSMC apoptosis is reduced in the 20-HETE and in the 20-HETE + HET0016 groups and increased in the HET0016 group, independently of how HUVSMCs were cultured.

of 20-HETE and HET0016 on cell proliferation using the MTT assay $(19,20)$. We found that $10 \mathrm{ng} / \mathrm{ml}$ of 20 -HETE and $10 \mu \mathrm{g} / \mathrm{ml}$ of HET0016 exerted the most prominent effect on the proliferation of HVTs and HUVSMCs after $48 \mathrm{~h}$ of treatment.

Our study found that 20-HETE can inhibit HVT invasion, while HET0016 can promote it. Numerous signal transduction pathways are involved in the regulation of HVT migration, among which the most important are HIF-1 $\alpha$ and PI3K/AKT/FOSL1 $(8,9,21)$. HIF-1 $\alpha$ can inhibit the migration of HVTs through promoting the expression of TGF- $\beta 3$, while PI3K/AKT/FOSL1 can enhance their ability to migrate through regulating the expression of downstream invasion-related proteins. Recent studies have found that 20-HETE is the upstream regulatory factor of signal transduction cascades, including HIF- $1 \alpha$ and PI3K/AKT/FOSL1, and through these signaling cascades, 20-HETE is able to regulate biological behaviors such as vascular cell proliferation and migration (9). These studies overall suggest that 20-HETE can inhibit the invasion of HVTs through a number of signal transduction pathways. The invasion of HVTs comprises two processes, namely migration and infiltration. To the best of our knowledge, there is no study reporting the effect of 20-HETE on the invasive ability of HVTs to date.

The degradation of the extracellular matrix of uterine spiral arteries is the main step in trophoblast invasion and spiral artery remodeling. A recent study showed that MMP-2 is an important molecule involved in the degradation of the extracellular matrix of uterine spiral arteries (14). In order to further explore the effect of 20-HETE on trophoblast invasion, 20-HETE and HET0016 were here used to treat HVTs in vitro. The results showed that 20-HETE has inhibitory effects on the transcription and the translation of the protein MMP-2 in HVTs, while HET0016 exhibited an enhancing effect. Under normal physiological conditions, the activation or inhibition of the transcription of endogenous MMPs allows to adjust their relative activities (22), while the decreased expression of placenta MMPs (MMP-2) leads to the relatively shallow invasion of HVTs and to spiral artery remodeling disorder (23). Therefore, the inhibition of the expression of MMP-2 by 20-HETE in HVTs might affect their ability to invade the uterine spiral arteries. In patients with early PE, the expression of AA metabolites (20-HETE) in the decidua was found to be significantly enhanced, and the MMP-2 levels in plasma and amniotic fluid were significantly increased $(23,24)$, indicating that metabolic abnormalities of 20-HETE and MMP-2 may underlie the remodeling disorders associated with PE. The present study is the first to report that 20-HETE can inhibit the invasion of HVTs, which might provide a new target in the investigation of therapies for spiral artery remodeling disorders and related pregnancy complications.

Our study also found that 20-HETE can inhibit the apoptosis of HUVSMCs, and strongly inhibit their apoptosis when they are co-cultured with HVTs, while HET0016 showed a promoting effect on apoptosis. To the best of our knowledge, this is the first time that an inhibitory role on HUVSMC apoptosis is demonstrated for 20-HETE. The extracellular death receptor pathway is the mediator of HVT-induced apoptosis of spiral arterial endothelial cells and smooth muscle cells (25-27). HVTs can express certain factors (TNF- $\alpha$, FasL and TRAIL) that bind to their relative receptors and activate caspase-3, thereby inducing apoptosis (6). A recent study found that 20-HETE is involved in cell apoptosis by adjusting the activity of caspase-3 (10). It was hypothesized that 20-HETE may inhibit apoptosis of HUVSMCs via regulating caspase-3, but this hypothesis on the underlying mechanism was not confirmed.

In addition, our study revealed that the combination of 20-HETE with HET0016 surprisingly exerts similar, and even stronger, effects compared to treatment with 20-HETE alone. A comprehensive review of the current literature suggested that: HET0016 selectively inhibits the de novo synthesis of 20-HETE by targeting CYP 4A and 4F, while it can not inhibit the activity of total free 20-HETE in vivo, and polymorphisms in the $C Y P 4 A$ and $C Y P 4 F$ genes may result in related functional changes (28-30). Another type of AA metabolite, EETs, was also found to be involved in vascular protection, anti-inflammatory and renal excretion, and in endogenous compression. Under normal physiological conditions, HVTs, the placenta, amnion, decidua and gravid myometrium cells all express EETs (31). The in vivo secretion of 20-HETE and EETs is regulated by independent isozymes, while their biological activities intertwine, so that 20-HETE and EETs can both regulate the fluid balance through inhibiting relative ion 
channels and the PI3K/AKT signaling pathway $(12,15,31,32)$. In our experiments, a potential feedback effect of 20-HETE and HET0016 on EETs was not explored. HET0016 could not inhibit the activity of total free 20-HETE in vivo; the high concentration HET0016 could not only inhibit the synthesis of 20-HETE, but also inhibit the synthesis of consecutive products of arachidonic acid (29). Along the course of development of 20-HETE inhibitors, which started with 17-ODYA, and eventually led to 1-ABT and HET0016, the specificity of the inhibition gradually increased $(13,17,30)$. Since the metabolites of AA are mainly isomers, the development of inhibitors has presented certain difficulties, and thus the specificity of HET0016 still requires further verification.

The results from the present study confirmed that 20-HETE can inhibit the invasion and apoptosis of HVTs, and strongly inhibit the apoptosis of HUVSMCs when co-cultured with HVTs. HVT and HUVSMCs are the main cells involved in USAR. During the remodeling process, the invasive ability of HVTs and the apoptosis of HUVSMCs are altered, which leads to the remodeling disorder, and indicates that abnormalities in the 20-HETE metabolism in the placenta uterina may lead to the spiral arteries remodeling disorder. At the early stages of pregnancy, USAR disorders are centrally associated with the occurrence of PE. Further investigation on the mechanism of action of 20-HETE with regards to the reported effects on the biological behavior of HVTs and HUVSMCs, may provide new clues for the etiology and pathophysiology of PE.

It should be noted that, due to sampling difficulties, research on USAR has its limitations (5). Here, in order to study spiral artery remodeling, we used uterine vascular smooth muscle cells instead of spiral arterial smooth muscle cells. It is generally established from previous studies that the biological functions of uterine arterial and spiral arterial smooth muscle cells are similar $(5,6)$.

In conclusion, as an upstream regulatory factor in multiple signal transduction pathways involved in the remodeling process and in vascular maintenance, 20-HETE can effectively inhibit the invasion of HVTs and strongly inhibit the apoptosis of uterine smooth muscle cells co-cultured with HVTs. This indicates that abnormalities in the metabolism of 20-HETE cause inaccurate transduction of the associated signals, which may lead to USAR and vascular functional disorders, and eventually cause PE.

\section{References}

1. Shennan AH, Redman C, Cooper C and Milne F: Are most maternal deaths from pre-eclampsia avoidable? Lancet 379: 1686-1687, 2012.

2. Young BC, Levine RJ and Karumanchi SA: Pathogenesis of preeclampsia. Annu Rev Pathol 5: 173-192, 2010.

3. Brosens I, Robertson WB and Dixon HG: The physiological response of the vessels of the placental bed to normal pregnancy. J Pathol Bacteriol 93: 569-579, 1967.

4. Harris LK: Review: trophoblast-vascular cell interations in early pregnancy: how to remodel a vessel. Placenta 31: S93-S98, 2010.

5. Pijnenborg R, Vercruysse L and Hanssens M: The uterine spiral arteries in human pregnancy: facts and controversies. Placenta 27: 939-958, 2006.

6. Whitley GS and Cartwright JE: Cellular and molecular regulation of spiral artery remodelling: lessons from the cardiovascular field. Placenta 31: 465-474, 2010.

7. Bulmer JN, Innes BA, Levey J, et al: The role of vascular smooth muscle cell apoptosis and migration during uterine spiral artery remodeling in normal human pregnancy. FASEB J 26: 2975-2985, 2012.
8. Guo AM, Scicli G, Sheng J, Falck JC, Edwards PA and Scicli AG: 20-HETE can act as a nonhypoxic regulator of HIF-1alpha in human microvascular endothelial cells. Am J Physiol Heart Circ Physiol 297: H602-H613, 2009.

9. Chen L, Ackerman R and Guo AM: 20-HETE in neovascularization. Prostaglandins Other Lipid Mediat 98: 63-68, 2012.

10. Dhanasekaran A, Bodiga S, Gruenloh S, et al: 20-HETE increases survival and decreases apoptosis in pulmonary arteries and pulmonary artery endothelial cells. Am J Physiol Heart Circ Physiol 296: H777-H786, 2009.

11. Pat Kunert M and Drenjancević I: 20-hydroxyeicosatetraenoic acid, endothelial dysfunction and hypertension. Med Glas (Zenica) 8: 170-180, 2011.

12. Llinás MT, Alexander BT, Capparelli MF, Carroll MA and Granger JP: Cytochrome P-450 inhibition attenuates hypertension induced by reductions in uterine perfusion pressure in pregnant rats. Hypertension 43: 623-628, 2004.

13. Stec DE, Gannon KP, Beaird JS and Drummond HA: 20-Hydroxyeicosatetraenoic acid (20-HETE) stimulates migration of vascular smooth muscle cells. Cell Physiol Biochem 19: 121-128, 2007.

14. Hazan AD, Smith SD, Jones RL, Whittle W, Lye SJ and Dunk CE: Vascular-leukocyte interactions: mechanisms of human decidual spiral artery remodeling in vitro. Am J Pathol 177: 1017-1030, 2010.

15. McGiff JC and Quilley J: 20-HETE and the kidney: resolution of old problems and new beginnings. Am J Physiol 277: R607-R623, 1999.

16. Alexanian A and Sorokin A: Targeting 20-HETE producing enzymes in cancer - rationale, pharmacology, and clinical potential. Onco Targets Ther 6: 243-255, 2013.

17. Guengerich FP and Rendic S: Update information on drug metabolism systems-2009, part I. Curr Drug Metab 11: 1-3, 2010.

18. Miyata N, Taniguchi K, Seki T, et al: HET0016, a potent and selective inhibitor of 20-HETE synthesizing enzyme. Br J Pharmacol 133: 325-329, 2001.

19. Wang Z, Tang X, Li Y, et al: 20-Hydroxyeicosatetraenoic acid inhibits the apoptotic responses in pulmonary artery smooth muscle cells. Eur J Pharmacol 588: 9-17, 2008.

20. Bodiga S, Gruenloh SK, Gao Y, et al: 20-HETE-induced nitric oxide production in pulmonary artery endothelial cells is mediated by NADPH oxidase, $\mathrm{H}_{2} \mathrm{O}_{2}$, and PI3-kinase/Akt. Am J Physiol Lung Cell and Mol Physiol 298: L564-L574, 2010.

21. Soares MJ, Chakraborty D, Renaud SJ, et al: Regulatory pathways controlling the endovascular invasive trophoblast cell lineage. J Reprod Dev 58: 283-287, 2012.

22. Lambert E, Dassé E, Haye B and Petitfrère E: TIMPs as multifacial proteins. Crit Rev Oncol Hematol 49: 187-198, 2004.

23. Palei AC, Granger JP and Tanus-Santos JE: Matrix metalloproteinases as drug targets in preeclampsia. Curr Drug Targets 14: 325-334, 2013

24. Lavee M, Goldman S, Daniel-Spiegel E and Shalev E: Matrix metalloproteinase-2 is elevated in midtrimester amniotic fluid prior to the development of preeclampsia. Reprod Biol Endocrinol 7: 85, 2009.

25. Ashton SV, Whitley GS, Dash PR, et al: Uterine spiral artery remodeling involves endothelial apoptosis induced by extravillous trophoblasts through Fas/FasL interactions. Arterioscler Thromb Vasc Biol 25: 102-108, 2005.

26. Harris LK, Keogh RJ, Wareing M, et al: Invasive trophoblasts stimulate vascular smooth muscle cell apoptosis by a fas ligand-dependent mechanism. Am J Pathol 169: 1863-1874, 2006.

27. Stoneman VE and Bennett MR. Role of Fas/Fas-L in vascular cell apoptosis. J Cardiovasc Pharmacol 53: 100-108, 2009.

28. Peppiatt C and Attwell D: Neurobiology: feeding the brain. Nature 431: 137-138, 2004.

29. Hoff U, Lukitsch I, Chaykovska L, et al: Inhibition of 20-HETE synthesis and action protects the kidney from ischemia/reperfusion injury. Kidney Int 79: 57-65, 2011.

30. Wu CC and Schwartzman ML: The role of 20-HETE in androgen-mediated hypertension. Prostaglandins Other Lipid Mediat 96: 45-53, 2011.

31. Jiang H, McGiff JC, Fava C, et al: Maternal and fetal epoxyeicosatrienoic acids in normotensive and preeclamptic pregnancies. Am J Hypertens 26: 271-278, 2013.

32. Attwell D, Buchan AM, Charpak S, Lauritzen M, Macvicar BA and Newman EA: Glial and neuronal control of brain blood flow. Nature 468: 232-243, 2010. 